Universidad Nacional Experimental Francisco de Miranda (UNEFM). Santa Ana de Coro. Venezuela

Laura Verónica Riera-Peralta; Patricio Esteban Mendieta-Andrade; Priscila Elizabeth Muñoz-Castro

DOI $10.35381 / \mathrm{cm} \cdot v 6 \mathrm{i} 2.369$

\title{
Control de costos enfocado a la toma de decisiones en el sector de la construcción
}

\section{Cost control focused on decision making in the construction sector}

\author{
Laura Verónica Riera-Peralta \\ laura.riera24@est.ucacue.edu.ec \\ Universidad Católica de Cuenca, Cuenca \\ Ecuador \\ https://orcid.org/0000-0002-2133-3280 \\ Patricio Esteban Mendieta-Andrade \\ pmendietaa@ucacue.edu.ec \\ Universidad Católica de Cuenca, Cuenca \\ Ecuador \\ https://orcid.org/0000-0001-9596-4344 \\ Priscila Elizabeth Muñoz-Castro \\ pmunoz@ucacue.edu.ec \\ Universidad Católica de Cuenca, Cuenca \\ Ecuador \\ https://orcid.org/0000-0001-6260-4190
}

Recibido: 05 de agosto de 2020

Aprobado: 01 de octubre de 2020 


\title{
RESUMEN
}

Las empresas constructoras como ejes sustanciales en la economía del Ecuador requieren de procesos contables que garanticen la rentabilidad y crecimiento, abordando de esta manera los fundamentos de los costos de producción en la aplicación de un control de costos en este sector, surgiendo como problema la falta de estimación real de los mismos, lo que imposibilita mitigar los riesgos que pueden afectar la estabilidad económica de la empresa, en consecuencia, se establece como objetivo elaborar un control de costos en el marco del enfoque por actividades $A B C$ que afiance los resultados de manera íntegra y sistematizada, para tal efecto, la investigación se sustentó en el alcance descriptivo que permitió determinar el deficiente cálculo de los costos en proyectos de construcción a causa del desconocimiento por parte de los profesionales contables, concluyendo en la necesidad de implementar un proceso efectivo de control de costos.

Descriptores: Contabilidad; costes; industria; empleo; producción. (Palabras tomadas del Tesauro UNESCO).

\begin{abstract}
Construction companies as substantial axes in the economy of Ecuador require accounting processes that guarantee profitability and growth, thus addressing the fundamentals of production costs in the application of cost control in this sector, emerging as a problem the lack of real estimate of them, which makes it impossible to mitigate the risks that may affect the economic stability of the company, consequently, The objective is to develop a cost control within the framework of the ABC approach that consolidates the results in an integral and systematized manner. To this end, the research was based on the descriptive that allowed to determine the deficient calculation of the costs in construction projects due to the lack of knowledge on the part of the accounting professionals, concluding in the need to implement an effective cost control process.
\end{abstract}

Descriptors: Accounting; costs; industry; employment; production. (Words taken from the UNESCO Thesaurus). 
Laura Verónica Riera-Peralta; Patricio Esteban Mendieta-Andrade; Priscila Elizabeth Muñoz-Castro

\section{INTRODUCCIÓN}

La contabilidad es un mecanismo fundamental de amplio espectro para el desarrollo de toda actividad económica de la sociedad, por lo que tiene una incidencia directa en la implementación de herramientas y técnicas vinculadas a esta disciplina, a efectos de obtener mejores resultados en cuanto a los datos y las cifras de una esfera socioeconómica se refiere, a través de un detalle meticuloso, preciso y sobre todo real adaptable a los cambios que se ocasionen tanto en el ámbito público como el privado conforme al control contable empleado al caso en concreto.

Bajo este contexto, la actividad contable implica un conjunto de operaciones económicas reflejadas en las cuentas de registro cuantificable que luego de un proceso sistemático son capaces de rendir información auténtica de la actividad económica y financiera de una empresa, la misma permite apreciar su condición de crecimiento como resultado de la gestión productiva y la posible toma de decisiones de modo estratégico con objeto de mejorar sus rendimientos financieros como expresión máxima del correcto funcionamiento de la empresa.

En consideración con lo expuesto, se requiere impulsar un control de los costos de producción en las operaciones económicas de las entidades del sector de la construcción, por ser considerada una de las actividades más dinámicas de una economía, esto permitirá el perfeccionamiento en la toma de decisiones administrativas a través de un contenido analítico y focalizado, por lo tanto, es fundamental la comprensión de la noción de costos de producción, puesto que, esta actividad no implica la consecución de procedimientos inclinados a determinar el costo de los servicios y productos, sino también, a liderar el mercado competitivo a través del crecimiento empresarial que, en lo referente a la ejecución de los proyectos del sector de la construcción de mediana y gran envergadura están ligadas a otros aspectos como la promoción del empleo, el impulso de la actividad económica, el beneficio de los productos primarios y demás elementos aplicables a este sector. 
Laura Verónica Riera-Peralta; Patricio Esteban Mendieta-Andrade; Priscila Elizabeth Muñoz-Castro

Por consiguiente, este trabajo sitúa el problema de investigación con enfoque a la estimación de los costos de producción de las empresas de construcción con la intención de mitigar los riesgos posibles de pérdidas que los afecten, pues esta falta de asignación produce una especie de alteración a la hora de la determinación económica, debido a las particularidades presentes en las distintas etapas de la construcción, en el empleo de los recursos asignados para el efecto, así como, el uso del material primario, como elementos constitutivos del producto final que debido al desconocimiento de su costo causan conmutación en los resultados contables.

Bajo estos argumentos, se formula el siguiente problema de investigación: ¿cómo determinar la estimación correcta de costos en el proceso productivo de las empresas constructoras? Por consiguiente, el objetivo del presente estudio consiste en elaborar un control de costos en el marco del enfoque por actividades $A B C$ en las empresas constructoras para la estimación correcta de los costos de producción.

\section{Referencial teórico}

Fundamentos generales de los costos de producción y sus características en los proyectos del sector de la construcción

En un acercamiento a la contabilidad general la noción referente al costo de un servicio o producto será sistematizado como la valoración de los recursos económicos empleados para la consecución de un objetivo específico comprendido por la retribución de la mano de obra, el importe cancelado por los materiales usados para su fabricación, más la fracción de los costos generales de funcionamiento de la empresa o negocio como el alquiler o la depreciación de un edificio (según sea alquilado o propio), mantenimiento, electricidad, seguros, reparaciones, devaluación de los equipos y maquinarias, entre otros.

De ahí que, el costo es entendido como el esfuerzo o sacrificio económico que se realiza para lograr un objetivo, que entre otras situaciones, sea operativo como la compra de materiales, cancelación de sueldos, prestación de servicios, administración de la 
Laura Verónica Riera-Peralta; Patricio Esteban Mendieta-Andrade; Priscila Elizabeth Muñoz-Castro empresa, obtención de financiamiento, entre otros, tal como lo manifiestan autores como (Horngren, Srikant, \& Foster, 2007) que categorizan la contabilidad de costos como el mecanismo encaminado a establecer los componentes que permitan obtener resultados ya sea financieros o no, en atención a los costos operacionales como al implemento de los recursos de la empresa al referirse a esta clase de contabilidad hace hincapié de su importancia para la valoración financiera en la medida que los costos se presentan como situaciones relevantes para la consecución de los resultados financieros de una empresa. De donde se infiere que el control de costos también puede ser determinado como el conjunto de normas, métodos y procedimientos con la finalidad de satisfacer las necesidades de control, determinación, planificación y estudio del costo, así como, el procedimiento de registro de los gastos de las actividades productivas de una empresa, de modo interrelacionado con los subsistemas que avalan el control de la producción o servicio y de los recursos laborales, materiales y financieros, para la comprensión de los resultados alcanzados sobre la base de un presupuesto que permita la toma de decisiones según las diferentes alternativas y operaciones.

De ahí que, en las compañías dedicadas al rubro de la construcción, el principal objetivo del manejo estratégico de los costos sea la organización de la información, con el objetivo de facilitar en las empresas la conservación de su competitividad y el mejoramiento diario de sus productos y servicios, pues el reflejo de este proceso de información contable les permitirá con fundamento en la Norma Internacional de Contabilidad (NIC 11) preparar informes de control de los costos e ingresos de los contratos de construcción, en los que, se distribuyan los ingresos ordinarios y los costos relacionados con ellos entre los periodos contables en los que se ejecutan, todo esto a partir de los criterios determinados en el marco conceptual para la preparación y presentación de los estados financieros, con la identificación de los costos que han influido en las actividades del proceso productivo con capacidad de brindar información confiable (Mogrovejo-Juela, NarváezZurita, \& Erazo-Álvarez, 2019). 
Laura Verónica Riera-Peralta; Patricio Esteban Mendieta-Andrade; Priscila Elizabeth Muñoz-Castro

Sin embargo, en este punto es necesario recalcar que si se considera que en el sector de la construcción existen diferencias entre el modo de registrar los costos futuros de una obra durante su fase de inicio y los cortos reales en los que incurre la empresa, con el objeto de emprender la comparación de la información entre el costo real y los costos ocasionados en la fase de ejecución y diseño, deberán incorporarse a los costos imputables a un contrato de construcción, además de los costos directos relacionados con el contrato específico, los costos que se imputen al acto contractual en general que puedan diferenciarse de otros contratos específicos, así como, los que conciernan a las actividades de la empresa en general o relacionados con el acto contractual que no pueden atribuirse entre contratos específicos.

Es así que, en los costos relacionados con el contrato específico deberán incluirse situaciones relativas a los principales recursos que se empleen para colocar de manera física los elementos de construcción en el proyecto, tales como: los costos de la mano de obra en el lugar de la construcción, salario y cualquier otro gasto referente a este concepto (entendido como el esfuerzo mental o físico utilizado para la fabricación del producto), la depreciación de los equipos, maquinarias, herramientas y, demás usados en el desarrollo del contrato, las subcontrataciones, los materiales utilizados para la construcción presupuestada y cualquier otro costos relacionado con el traslado de la planta y equipo al sitio de la construcción que permiten determinar los costos que estarán presentes siempre en la actividad constructiva y, los costos variables que fluctúan a partir de la particularidad del proceso productivo (Galarza-Pomaquiza, Narváez-Zurita, \& Erazo-Álvarez, 2019).

Mientras que, en lo referente a los costos que pueden atribuirse al acto contractual en general y pueden diferenciarse de otros contratos específicos, deberán incluirse ciertos patrones concernientes con el diseño y asistencia técnica, seguros o gastos indirectos de construcción y, en los costos relativos a las actividades de la empresa en general o relacionados con el acto contractual que no pueden atribuirse entre contratos específicos se deberá señalar los costos de financiamiento, investigación, desarrollo, generales de 
Laura Verónica Riera-Peralta; Patricio Esteban Mendieta-Andrade; Priscila Elizabeth Muñoz-Castro

administración y ventas, depreciación de planta y equipo que no se usan en un contrato particular.

Debiendo recalcarse que los equipos, maquinarias, herramientas e instalaciones que dan apoyo al proceso productivo y sus costos señalados en los párrafos anteriores, pueden ser divididos en dos grupos, en la medida que el activo sea de propiedad de la empresa, donde los costos estarán conformados por el concepto de depreciación del valor por el uso, el desuso y desgate o si el activo es arrendado, donde el costo que deberá imputarse a la obra será el pago por arrendamiento, en tal razón, el control de costes maneja estrategias particulares en la gestión del costo de las empresas constructoras apropiadas para tomar las decisiones necesarias en el proceso productivo (Lindao-Bravo, Narváez-Zurita, \& Erazo-Álvarez, 2019).

Todo esto demuestra el control de costos, como un punto crítico a la hora de satisfacer las necesidades de control de las empresas de construcción, debido a que sus controles de acumulación tienen un gran influjo en sus resoluciones, crecimiento y supervivencia, por cuanto, a través del mismo se computan los costos de producción, se valoran los inventarios, las utilidades, se pueden fijar precios y descuentos de cada producto, se conocen los productos más rentables, entre otros rubros que no son manejados en la orientación correcta, contable y administrativa; el desconocimiento de los actos ejecutados con dichos costos puede ocasionar un desequilibrio del balance de la empresa.

Por consiguiente, resalta la necesidad de su utilización por parte de la administración de las compañías del sector de la construcción, al conformarse en una base indispensable para el control, planificación y adopción de sus decisiones y demás fases del proceso general contable, a pesar de que según los datos proporcionados por la contabilidad de costos, frente a la inestabilidad macroeconómica y en general el contexto económico del Ecuador, no garantizan el éxito de las resoluciones tomadas, ya que todo dependerá del modo como se muestre y se examine la información. 
En los controles de información financiera, la aplicación de un modelo de gestión de costos se presenta como un instrumento de gran valor inmerso en las estructuras empresariales que lo emplean, de esta manera, se pone en evidencia la relevancia del control de costos en las empresas, al ser una contabilidad útil para la fase de adopción de resoluciones y para la obtención de información confiable, oportuna y relevante, pues a través de ella las empresas que operen mediante la variable costos, podrán emplear además estos factores como indicadores capaces de facilitar la competencia y la gestión de proyectos.

Considerando la trascendencia que las empresas constructoras han tenido en el desarrollo económico y social del Ecuador, así como, su capacidad para crear empleo, en las siguientes líneas se examina la importancia de la utilización de un registro de información apoyado en la gestión de costos de producción, mediante un control de acumulación por actividades adecuadas a la realidad de la organización, que permita a las empresas de este sector, no solo, consolidar un proceso contable en sentido confiable, sino también a partir de la comparación de sus costos reales y ocasionados en la fase de ejecución y diseño de sus productos, proporcionar datos adecuados para la adopción de decisiones y el mejoramiento de la gestión empresarial.

\section{Aplicación del control de costos basado en actividades en los procesos productivos de la industria constructora}

El control de costos fundamentado en actividades, denominado $A B C$ por sus siglas en inglés derivadas de la expresión anglosajona Activity Based Costing, fue incorporado a finales de la década de los ochenta, como novedoso mecanismo de gestión dirigido a mejorar el poder de análisis en la valoración de los costos de los servicios y productos generados por una empresa, así como, reducir e identificar las actividades que no prestan un valor agregado, por lo que, dadas sus ventajas relativas al control de la dirección organizativa y la valoración de las decisiones que pudieran ser adoptadas en esta estructura, en su desarrollo será categorizado como un instrumento importante de 
Laura Verónica Riera-Peralta; Patricio Esteban Mendieta-Andrade; Priscila Elizabeth Muñoz-Castro

análisis para tomar oportunas decisiones frente a las formas tradicionales de asignación de costos.

Para complementar a estas consideraciones, el control $A B C$ se fundamenta en operaciones que facilitan una percepción integra de los costos de la empresa al administrar el valor de los procesos, de las actividades y del consumo de los recursos (Viamontes, Utra, \& Cruz, 2000). Para tal efecto, se asigna de manera metodológica todos los costos indirectos generados por la empresa a sus actividades con relación al producto que desarrolla, para luego dividir el alcance de los costos y el consumo de las actividades operativas, entre sus productos y servicios, de esta manera, guía la obtención de información valiosa para la supresión de los costos que no generan ningún valor significativo a la empresa constructora, a fin de conseguir perfeccionamiento continuó de la organización y un mejor aprovechamiento en los recursos.

La concepción relativa a la cadena de valor, impone también la exigencia de efectuar un estudio desarticulado de costos, sin perder de vista las particularidades del ejercicio diario de la empresa, donde la unidad relevante para este examen estratégico recae en las actividades que consumen los recursos y no los productos ni servicios concluidos, pues emplear un control de costos a nivel de unidad de obra, presupondría un parámetro para un estudio demasiado limitado que impediría la obtención de conclusiones significativas desde un factor de competitividad, en el sentido que, si una actividad llevada a cabo es consustancial a diferentes productos, los datos trascendentes en materia de costos no son los que provienen de cada uno de ellos, sino los que pueden ser derivados del resultado procedente por todos los productos y servicios que participan en la actividad. En otras palabras, en opinión de los autores (Catalá-Alís \& Yepes-Piqueras, 1999) el control de gestión $\mathrm{ABC}$ consistirá en incorporar al costeo, una medida de evaluación de desempeño estructurado no por el margen de ventas, sino por el empleo que cada actividad específica se haga en cada producto, razón por la que, la asignación de los costos por lo general se ejecuta a partir de un análisis compuesto por tres dimensiones (costo, tiempo y calidad), dividido en dos etapas diferenciadas, concernientes, por un 
Laura Verónica Riera-Peralta; Patricio Esteban Mendieta-Andrade; Priscila Elizabeth Muñoz-Castro

lado, a los costos que no pueden ser imputados a cada unidad de obra que a su vez pueden ser incorporados en los costos que posean un vínculo común, y por otro lado, a la selección de los mecanismos de asignación de gastos de cada una de las unidades de obra a través de sus conexiones causales que proyectan la ejecución de las actividades. Por consiguiente, cada actividad diferenciada, puede tener su propio generador o inductor de costos, definidos como todos aquellos elementos esenciales que facilitan la determinación del comportamiento del costo de una actividad, a través de la valoración del costo de elaboración de un producto o servicio inmerso en la cadena de valor de un negocio (Rodríguez-Mañay, Saltos-Chacán, \& Muñoz-Moreta, 2016). De ahí que, una definición de la cadena de valor como: "(...) el conjunto de actividades creadoras de valor" (Torres- Salinas, 2010, p. 27), reforzará esta idea relativa a la necesidad de un análisis desagregado de los costos de cada actividad y de la selección de los criterios de reparto, en la medida que, un estudio de esta naturaleza servirá también de aporte para la delimitación del trayecto estratégico y el examen de las actividades empresariales con la finalidad de visibilizar las fuentes posibles de ventaja competitiva.

Razón por la que, habiéndose definido la cadena de valor como el conjunto de actividades principales como secundarias de la actividad empresarial, siguiendo las investigaciones realizadas por los autores (Hicks, 1998); (Kaplan \& Cooper, 2000); (Cuevas, Chavez, Castillo, Caicedo, \& Solarte, 2004); (Ortega-Godoy, 2006); (Guerra \& Yero, 2009) y (Arias-Cubillo \& López-López, 2010), se asume que la asignación de los costos se deberá realizar mediante un análisis que aplique los procedimientos de la contabilidad de gerencia cimentada en actividades y la selección de sus criterios de reparto que pueden ser resumidos en los pasos siguientes:

1. Individualizar las diversas actividades relevantes de la cadena de valor.

2. Sistematizar los inductores o generadores de costos como mecanismos de cuantificación del volumen de costos relacionados con cada actividad.

3. Asociar las actividades homogéneas que se desplieguen en los centros de responsabilidad y cálculo del costo unitario del generador o inductor de costos. 
Laura Verónica Riera-Peralta; Patricio Esteban Mendieta-Andrade; Priscila Elizabeth Muñoz-Castro

4. Asignar del costo del servicio o producto final a través de la incorporación de los costos fijados a los elementos intermedios que lo componen.

De lo expuesto, se demuestra que el tratamiento metodológico de un control de gestión $\mathrm{ABC}$ para el sector de la construcción, no implica una imputación lineal arbitraria, puesto que la fijación de los costos se deberá cimentar en las actividades ejecutadas para la elaboración de cada unidad de obra, puesto que la implementación de un control $A B C$ debe configurarse como mínimo a partir de cuatro categorías diferentes de clases de actividades, que siguiendo los criterios expuestos por los tratadistas (Guerra \& Yero, 2009) pueden resumirse en las siguientes:

1. Actividades primarias o de nivel unitario que son aquellas que se efectúan cada vez que se elabora o desarrollar una unidad de producto.

2. Actividades de nivel lote que se ejecutan cada vez que una fracción de productos es elaborada.

3. Actividades de mantenimiento que se materializan como un requerimiento para sostener la producción de cada clase de producto.

4. Actividades de soporte o apoyo que mantienen la ejecución general del procedimiento de elaboración.

Debe tenerse en cuenta que en los tres primeros rangos es factible imputar los costos ocasionados a cada una de las respectivas unidades de obra, mientras que, en lo que respecta a las actividades de soporte como las referentes a la planificación, gestión de calidad, seguridad y limpieza de la infraestructura, la compra de materia prima, maquinaria, suministros, artículos consumibles, investigación y desarrollo, acciones de naturaleza administrativa entre otras, se deberá efectuar una fijación de costos para frenar en lo posible una asignación arbitraria a las unidades que, incluso bajo ciertas circunstancias debería evitarse cuando no incorpore información económica que facilite la aplicación de acciones correctivas para dicha unidad.

Además debe mencionarse que los costos fijados en el control $A B C$, no se ajustan a los determinados en el modo tradicional, en la medida que al suprimirse ciertos criterios, no 
solo se logra una menor valoración de los costos reales contenidos en cada unidad de obra, sino también, la obtención de datos de mejor calidad para la gestión de las empresas (estén o no ubicadas en el sector de la construcción), pues este control además de buscar la clasificación de las actividades que de acuerdo con (Brimson, 1997) pueden ser diferenciadas en directas, indirectas y de garantía de la calidad, indaga también en cada una de ellas sobre los factores que impulsan su costo, de ahí la necesidad de una fijación rigurosa de los costos no imputables a cada unidad de obra, con la finalidad de evitar mediante la compensación de costos entre las diversas unidades, el ocultamiento de ineficiencias de las empresas constructoras y el desaprovechamiento de las oportunidades de mejoramiento de la competitividad.

\section{Efectos de la falta de un control de los costos de producción basado en actividades en el sector de la construcción}

Con la finalidad de analizar los costos de producción en el sector de la construcción, así como, las variables que se interrelacionan con el éxito o fracaso de su diseño e implementación, es necesario en primer lugar mencionar que la preocupación de las constructoras por el manejo de los costos, se originó como consecuencia del incremento de las presiones competitivas a las que se expone la industria de la construcción, según los autores (Arcudia-Abad, Pech-Pérez, \& Álvarez-Romero, 2005); (Ordoñez-Coronado, 2012); (Maldonado-Borja, 2017) y (Chang-Saltos, 2017) se evidencia de manera especial en aquellos países que se encuentran en vías de desarrollo que no han dejado de perseguir las estrategias del sector público, a pesar de que su actividad debería basarse de forma primordial en los estándares internacionales de construcción independiente de que se construyan para el régimen público o privado.

Bajo el contexto, el aumento de los requerimientos de la infraestructura civil, han obligado a las empresas a maniobrar en niveles cada vez mayores, presionando las operaciones y las capacidades administrativas de la industria de la construcción, a pesar de que no persigan con exactitud los modelos convencionales de la industria de proceso. De ahí 
Laura Verónica Riera-Peralta; Patricio Esteban Mendieta-Andrade; Priscila Elizabeth Muñoz-Castro que, en lo referente a las actividades de las compañías de la construcción empujadas por altos niveles de producción, investigadores como (Arcudia-Abad, Pech-Pérez, \& ÁlvarezRomero, 2005) mencionen la obligación de tener en cuenta en la administración de estas empresas, el manejo de tres acciones esenciales para una producción equilibrada y equivalente, divididas en las funciones de finanzas, operaciones y mercadeo, donde:

La función finanzas se encarga de asegurar a precios favorables los recursos. También se encarga de la evaluación de los proyectos que se pretenden ejecutar, analizando su factibilidad económica antes de invertir. (...) Las operaciones son parte medular de las funciones de la empresa, pues mediante ellas se crean los productos y servicios que suministran al entorno. (...) Mediante el mercadeo se promueve productos y servicios de la empresa, así como su venta a los clientes del ramo. (p. 28)

De ahí que, autores como (Noguera-López, 2005) analicen la función de finanzas y la preocupación de las constructoras por el manejo de los costos, exteriorizando esta preocupación de la administración de las empresas de construcción, al considerar que las mismas no admiten la ejecución de proyecciones adecuadas cuando los niveles de producción tienen fluctuaciones significativas. Situación que ha generado que las empresas de la construcción no posean un control de contabilidad de costos adecuado, en la medida que la generalidad de compañía si llevan algún registro financiero esencial para fines fiscales, estos datos resultan insuficientes para determinar que productos, trabajos o servicios son los más rentables.

Debiendo recalcarse en este punto, conforme las investigaciones realizadas por (Sierra, 2002); (Morillo-Moreno, 2003); (González-Pesantez \& Muñoz-Delgado, 2010); (SalinasBonilla, 2012) y (García, Galo, \& Villafuerte, 2015) que casi la totalidad de las empresas de la construcción ubicadas en el Ecuador no conocen la estructura de costos mediante controles de acumulación ya sea por productos, procesos, actividades u otro criterio adecuado a la realidad de la organización, ni la situación financiera de cada uno de sus productos, ya que sus conocimientos insuficientes sobre los costos de producción, son calculados sin considerar los aspectos básicos y principios de esta clase de contabilidad, 
Laura Verónica Riera-Peralta; Patricio Esteban Mendieta-Andrade; Priscila Elizabeth Muñoz-Castro

lo que impide tener una visión clara de la situación financiera general de la empresa, pues los indicadores más significativos los consiguen de la contabilidad tradicional y algunas de las propias valoraciones de obra.

Razón por la que, está insuficiente organización en el tratamiento de los costos y la ausencia de un control de información que sirva de soporte para su gestión, hacen imperiosa la necesidad de incorporar a la administración de las empresas, un control que basado en actividades contribuya en la toma de resoluciones estratégicas de la organización, pues el desconocimiento de los costos trae como consecuencia, no solo, la inobservancia de los costos asociados a los equipos, servicios, mantenimiento, personal, dotación e insumo por actividades y de cada elemento empleado en una construcción que de forma eventual puede impedir el cálculo de la utilidad, sino también, un nivel bajo de competitividad, en el sentido que se desaprovecha el poder de negociación que puede otorgar el hecho de conocer los límites máximos de lo que se puede gastar al ofertar un producto o servicio, así como, la cuantía mínima que debe cancelar el usuario, con el objeto de que no se originen pérdidas (Cuevas, Chavez, Castillo, Caicedo, \& Solarte, 2004).

De ahí surge la necesidad de contar con un control adecuado de costos, que cimentado en actividades posibilite la asignación exacta de los costos de los productos y servicios que oferta una compañía de la construcción, así como, la rentabilidad de estos, siendo indispensable para el logro de este objetivo el conocimiento que se puede alcanzar, no solo, de los recursos e insumo que se demandan, sino también, de las secciones conexas a ellos, así como, de las actividades que los envuelven, para de esta manera, tener el control de la cantidad de materiales que se dispone y se requiere para la construcción, del personal indispensable para ejecutar el contrato y el presupuesto de los costos indirectos (Jiménez-Rodríguez \& Toala-García, 2014).

Por consiguiente, lleva a la necesidad de implantar mejoras en la gestión empresarial, con la finalidad de estructurar soluciones eficaces y oportunas a los desafíos de adaptación que exige un mercado globalizado, cada vez más severo, a través de 
resoluciones basadas en informes emitidos mediante la contabilidad de costos que en el presente análisis ha sido direccionado en un control de acumulación basado en actividades, donde el fracaso o éxito de la administración depende de la provisión exhaustiva de los datos relacionados con el valor de los procesos, actividades y el consumo de los recursos y no solo de los costos, ya que a través de una base oportuna y sistemática se pueden tomar decisiones relevantes para la gestión de la organización, así como, para el futuro de una empresa.

\section{MÉTODO}

La investigación se desarrolló bajo la finalidad transversal en atención a la recopilación de datos de contenido variable que caracteriza a la contabilidad de costos en el ámbito de la construcción, se estableció un alcance descriptivo para el logro de los objetivos propuestos, en consecuencia. Se estableció un muestreo por convivencia, basado en la técnica no aleatoria y muestreo no probabilístico mediante el empleo técnico de las encuestas, realizadas a los Gerentes del sector de la construcción en un número de 30 empresas, quienes respondieron a las preguntas planteadas en apego a las respuestas de opción múltiple en la escala líkert, establecidas para el efecto, con el propósito de fortalecer y contribuir con el proceso de la investigación, aplicándose estadística descriptiva.

\section{RESULTADOS}

Hoy en día, es indiscutible la necesidad de implementar una guía completa afianzada en el control de costos en el sector de la construcción que permita fomentar el crecimiento de la empresa con la implicación de procedimientos y técnicas que promuevan un análisis objetivo de su realidad a los resultados de la gestión administrativa empresarial, con el fin, de emplear estrategias inmediatas en su beneficio y, por lo tanto, contrarrestar los efectos negativos que pudiera incidir en su actividad. 
Laura Verónica Riera-Peralta; Patricio Esteban Mendieta-Andrade; Priscila Elizabeth Muñoz-Castro

En este sentido, el modelo de control de costos se enfoca en un mecanismo extraordinario de apoyo en el sostenimiento económico empresarial, mediante el manejo y control del proceso en la construcción, en contexto a la aplicación de la técnica abordada en esta investigación para los fines académicos se integra por encuestas que se llevaron a cabo con la participación de los representantes del sector de la construcción de la provincia del Azuay (Directivos-Administradores), de esta manera, se detallan a continuación los principales resultados más relevantes obtenidos:

\section{Información por el control de costos}

La importancia de establecer un control de costos es fundamental en la empresa, puesto que, revela información esencial de los resultados alcanzados, sin embargo, el 67\% de encuestados han señalado que de manera ocasional existe una información apropiada, mientras que, el $20 \%$ de los mismos ha indicado que casi todos los días tienen información adecuada y el $13 \%$ restante, todos los días, de los resultados económicos, al respecto, se evidencia la falta de información oportuna por los controles contables implementados en sus empresas.

\section{Control de costos en la empresa constructora}

Así mismo, el empleo de un control de costos de producción es fundamental en la empresa constructora, en este sentido, el $67 \%$ de encuestados ha determinado que de forma ocasional utiliza esta herramienta de verificación, mientras que, el $20 \%$ de los mismos lo emplea casi todos los días y en el $13 \%$ restante, es notoria la falta de implementación de un control de costos de producción en las empresas del sector de construcción.

\section{Registros individualizados de los costos}

Un manejo adecuado del control de costos es eficiente cuando se complementa con un registro particularizado del costo de las actividades que integran el proceso productivo en la construcción, por esta razón, para determinar si las empresas llevan un registro de sus costos, se ha visto la necesidad de efectuar una valoración con un rango moderado del 
1 al 5 , donde 1 implica una valoración baja en cuanto al uso de registro de costos y, 5 refleja una mayor ocupación del registro, en consecuencia, de los resultados obtenidos el $87 \%$ de encuestados ha valorado en el nivel uno, es decir, no tienen un registro de costos, en tanto que, el $7 \%$ restante ha valorado en el nivel dos y tres donde apenas emplean un prototipo de registro no tecnificado ni especificó para la descripción del costo, por lo tanto, las empresas carecen de un detalle adecuado de los costos que incide al momento de descifrar los indicadores del costo afectando el empleo de estrategias óptimas en beneficio de la empresa.

\section{Experiencia sobre el control de costos}

Esta situación traslada a poner vital atención sobre la experiencia del personal de la empresa sobre el control de costos de producción, contribuyendo a conseguir mejores resultados en el aprovechamiento instruido del control. De la situación descrita, el 87\% de encuestados considera que se encuentra poco satisfecha con la experiencia del personal responsable del control de costos, mientras que, el $13 \%$ restante está moderadamente satisfecha con el control de costos que domina su personal, en definitiva, en el marco del desarrollo e implementación del control de costos en la construcción, se requiere contar con personal que promueva el análisis y encauce la toma de acciones necesarias en la gestión de los costos de producción.

\section{Decisiones con base en el control de costos}

Para lograr el incremento del porcentaje de productividad en la empresa, se requiere admitir decisiones en simetría al control de costos de producción, para tal efecto, el $67 \%$ de encuestados ha coincidido que las decisiones de forma ocasional se basan en el control de costos, en tanto que, el $20 \%$ restante, casi todos los días, en la mayoría de casos no se adoptan las decisiones en concordancia a los costos. 
CIENCIAMATRIA

Revista Interdisciplinaria de Humanidades, Educación, Ciencia y Tecnología

Año VI. Vol. VI. N². Edición Especial II. 2020

Hecho el depósito de ley: pp201602FA4721

ISSN-L: 2542-3029; ISSN: 2610-802X

Universidad Nacional Experimental Francisco de Miranda (UNEFM). Santa Ana de Coro. Venezuela

Laura Verónica Riera-Peralta; Patricio Esteban Mendieta-Andrade; Priscila Elizabeth Muñoz-Castro

\section{PROPUESTA}

Una vez examinados los fundamentos teóricos del control de costos por actividades y, conforme la información recopilada mediante el diagnóstico, resalta la necesidad de implementar un control de costos $A B C$ en el sector de la construcción, por consiguiente, se propone la implementación de esta herramienta de acuerdo con el diagrama de la figura 1.

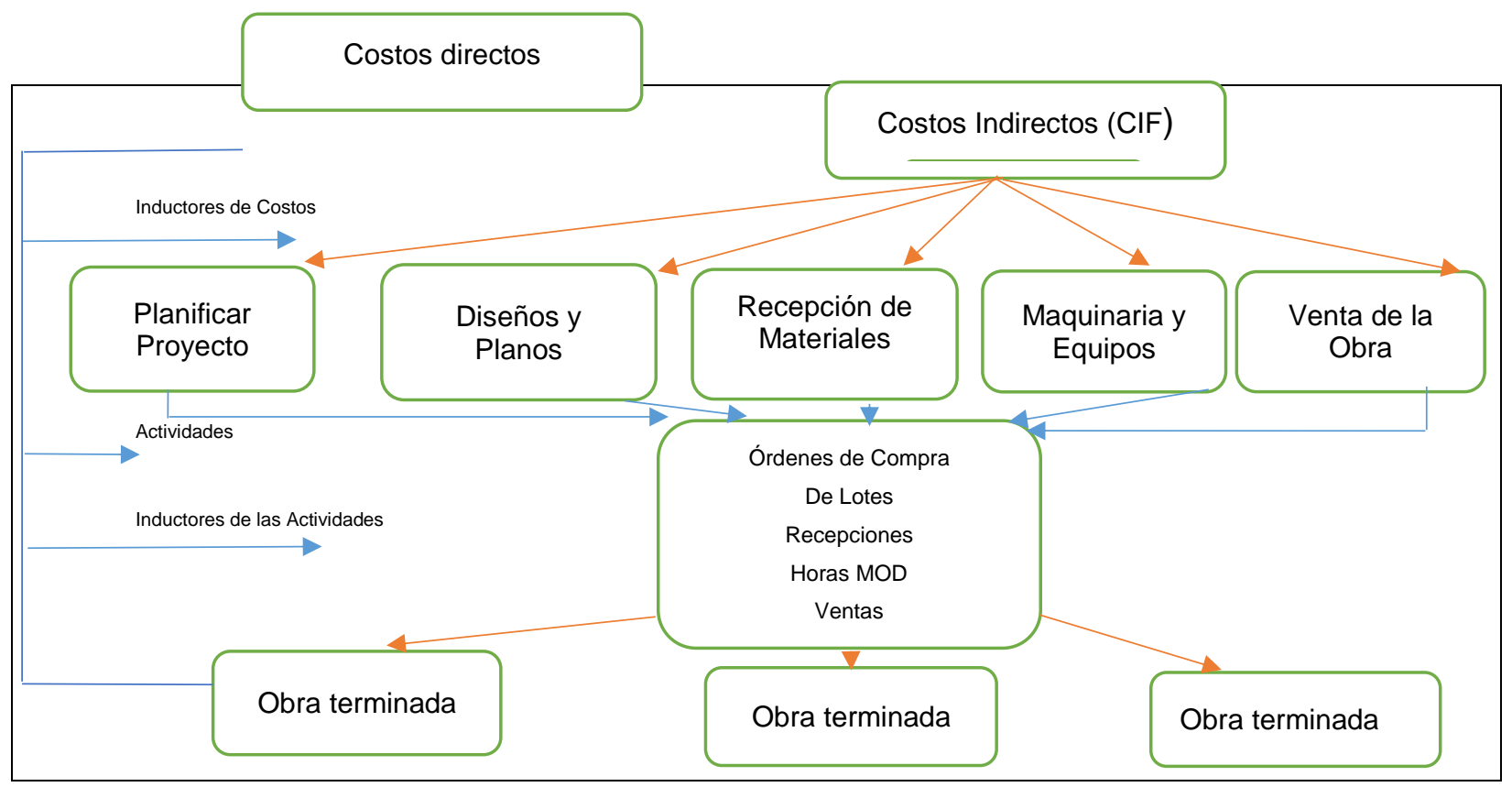

Figura 1: Diagrama de propuesta para un control de costos para la construcción.

En este sentido, de acuerdo con el esquema que antecede y al establecer lineamientos ajustados al proceso productivo relativos a la construcción, es menester determinar la razón de ser de la empresa que conforma la columna vertebral de la entidad a efectos de alcanzar sus fines, atendiendo a estas consideraciones, se establece como ejes primordiales de la empresa la postulación siguiente: 
Laura Verónica Riera-Peralta; Patricio Esteban Mendieta-Andrade; Priscila Elizabeth Muñoz-Castro

Misión: Creamos sitios de vivienda sustentables de manera estratégica y técnica acorde a las necesidades actuales que promuevan la erradicación de costos innecesarios y, de esta manera, alcanzar la vida digna del conglomerado social con incidencia positiva en la dinámica económica y en el desarrollo empresarial en el ámbito de la construcción.

Visión: Liderar el medio competitivo de la construcción nos llevará a la innovación de los procesos en los servicios atendiendo diversos estilos de vida de nuestros clientes alcanzando su respeto y confianza.

En esta perspectiva, se plantea el siguiente organigrama detallado en la figura 2:

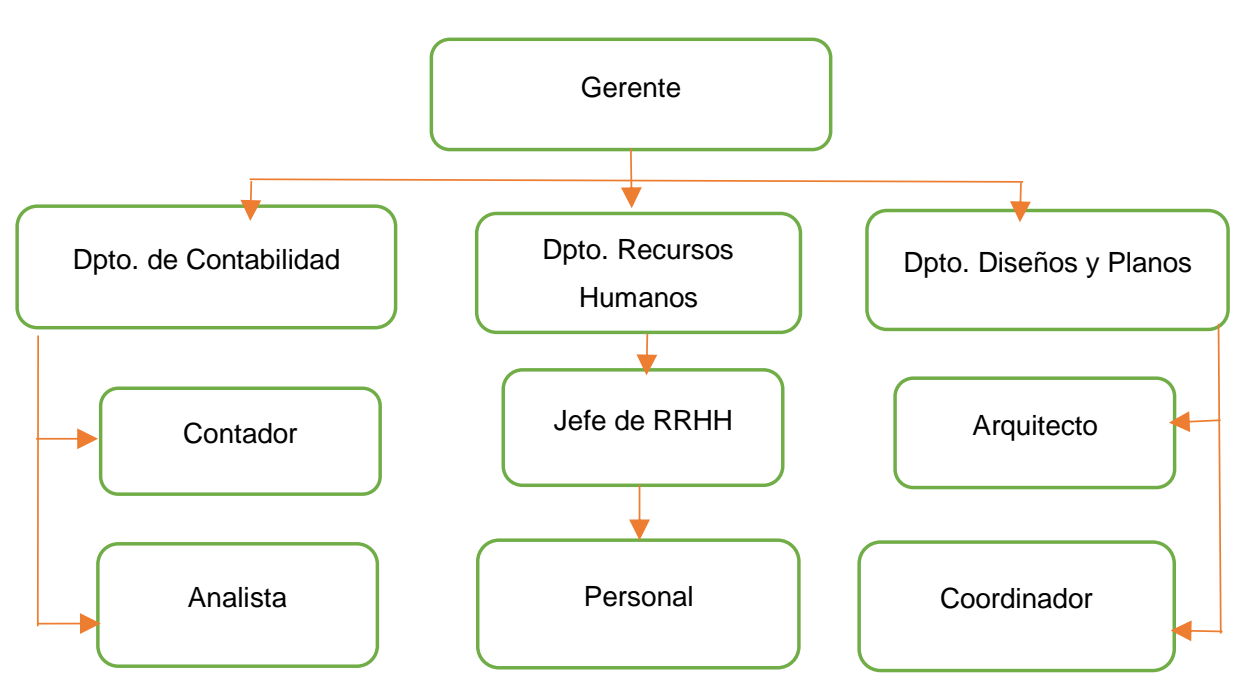

Figura 2: Organigrama de la empresa.

A este respecto, es importante la descripción de la obra idealizada para su edificación que se configura como una vivienda familiar urbana de características modernas habitable para cuatro personas en conformidad a los estándares de seguridad requeridas para este tipo de construcción con una dimensión de $90 \mathrm{~m}^{2}$; su repartimiento se especifica con una sala de $6 \mathrm{~m}^{2}$, una cocina de $14 \mathrm{~m}^{2}$, tres dormitorios de $10 \mathrm{~m}^{2}$ con baños interiores con $4 \mathrm{~m}^{2}$, el acceso residencial se ubica por la parte frontal de la casa, estas 
Laura Verónica Riera-Peralta; Patricio Esteban Mendieta-Andrade; Priscila Elizabeth Muñoz-Castro características básicas son adecuadas para el refugio de una familia cubriendo sus necesidades elementales además de proporcionarles privacidad y espacio suficiente.

\section{Determinación de la organización en la construcción}

En este sentido, para la materialización de la construcción se requiere la preselección de personal por talento humano, siendo el encargado de la dirección y procesos de selección a efectos de aprovisionar el contingente de mano de obra idónea y apta para el desarrollo de la construcción.

Así mismo, en apoyo a los fines de la construcción la logística cumple un papel importante sobre todo en lo que respecta a la cadena de abastecimiento, tendrá a su cargo la custodia, almacenamiento y control de los bienes con el fin de suministrar en las mejores condiciones los recursos necesarios para la ejecución de la obra.

Sobre esta conformación organizativa la empresa constructora al encontrarse circundado de actividades comerciales debe contar de manera imprescindible con la colaboración contable y administrativa que proporcione información precisa y oportuna en el desarrollo de sus actividades mediante la toma de decisiones apropiadas.

\section{Secuencia de la actividad constructiva}

Se expone el esquema de actividades en la construcción abarcando desde el proceso de inicio hasta la conclusión de la obra, detallado en la figura 3.

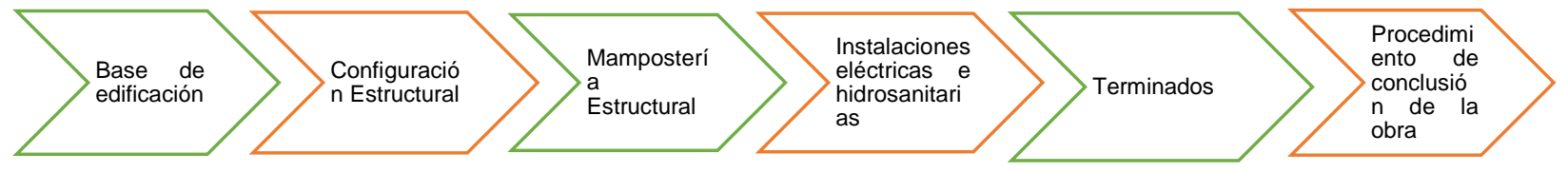

Figura 3. Secuencia de la actividad constructiva. 
Ahora bien, establecido el proceso de la construcción corresponde el momento de la determinación de los costos a cargar mediante la especificación de los centros de costo, la descripción de las actividades, los inductores de costos y encargados de la ejecución señalada en la tabla1.

\section{Tabla 1.}

Determinación de los costos.

\begin{tabular}{|c|c|c|c|}
\hline Centro de costos & Descripción & Inductores & Encargado de la Ejecución \\
\hline Planos diseño de la obra & Transformación & $\mathrm{H} /$ hombre & Arquitecto \\
\hline \multirow{6}{*}{ Construcción de la Obra } & $\begin{array}{c}\text { Aceptación } \\
\text { Modificación del Terreo } \\
\text { Modificación del Terreo } \\
\text { con el uso de maquinas }\end{array}$ & $\begin{array}{l}\text { H/hombre } \\
\text { H/hombre } \\
\text { H/máquina }\end{array}$ & $\begin{array}{l}\text { Obrero } \\
\text { Obrero }\end{array}$ \\
\hline & $\begin{array}{c}\text { Edificación de cimientos } \\
\text { Colocación de la estructura } \\
\text { Metálica }\end{array}$ & $\begin{array}{l}\mathrm{H} / \text { hombre } \\
\mathrm{H} / \text { hombre }\end{array}$ & $\begin{array}{l}\text { Obrero } \\
\text { Obrero }\end{array}$ \\
\hline & Ubicación de muros, paredes & $\mathrm{H} /$ hombre & Obrero \\
\hline & Instalaciones sanitarias & $\mathrm{H} /$ hombre & Plomero \\
\hline & Instalaciones eléctricas & $\mathrm{H} /$ hombre & Electricista \\
\hline & Terminados & $\mathrm{H} /$ hombre & Obrero \\
\hline
\end{tabular}

Luego de instaurar la determinación de los costos por actividades establecidos en el proceso de la construcción, llega el momento del análisis individualizado de los materiales directos e indirectos, así como la identificación del CIF, involucrados en el proceso de la construcción para dicho efecto se establece la tabla 2. 
CIENCIAMATRIA

Revista Interdisciplinaria de Humanidades, Educación, Ciencia y Tecnología

Año VI. Vol. VI. N². Edición Especial II. 2020

Hecho el depósito de ley: pp201602FA4721

ISSN-L: 2542-3029; ISSN: 2610-802X

Universidad Nacional Experimental Francisco de Miranda (UNEFM). Santa Ana de Coro. Venezuela

Laura Verónica Riera-Peralta; Patricio Esteban Mendieta-Andrade; Priscila Elizabeth Muñoz-Castro

Tabla 2.

Estudio de los costos por actividad.

Observación de los costos por actividad

Sección: Distribución de suministros de: Estructura metálica para la pared, base de tragaluces 2 conductos rectangulares de $25 \mathrm{~mm} \times 55 \mathrm{~mm} \times 1,5 \mathrm{~mm}$

Unidad: $\mathrm{m}$

Detalle: Contiene pintura antioxidante

\begin{tabular}{|c|c|c|c|c|c|}
\hline \multicolumn{6}{|c|}{ 1. Materiales } \\
\hline Detalle & Unidad & $\begin{array}{c}\text { Valor } \\
\text { A }\end{array}$ & $\begin{array}{l}\text { Precio Unitario } \\
\text { B }\end{array}$ & $\begin{array}{c}\text { Costo } \\
C=A \times B\end{array}$ & $\%$ \\
\hline $\begin{array}{l}\text { Conductor rectangular mecánico de } 25 \mathrm{~mm} \times 55 \mathrm{~mm} \times 1,5 \mathrm{~mm} \\
\text { Adherencia con Varilla WAS C-5022 }\end{array}$ & $\begin{array}{c}M \\
K G\end{array}$ & $\begin{array}{c}2 \\
0.25\end{array}$ & $\begin{array}{l}4.6429 \\
5.45\end{array}$ & $\begin{array}{l}9.29 \\
1.36\end{array}$ & $\begin{array}{r}43.01 \% \\
6.30 \%\end{array}$ \\
\hline
\end{tabular}

Subtotal W $49.31 \%$

\begin{tabular}{|c|c|c|c|c|c|c|}
\hline & & Mano de Obr & & & & \\
\hline Detalle & $\begin{array}{c}\text { Valor } \\
\text { A }\end{array}$ & $\begin{array}{c}\text { Salario/hora } \\
\text { B }\end{array}$ & $\begin{array}{l}\text { Valor hora } \\
\mathrm{C}=\mathrm{A} \times \mathrm{B}\end{array}$ & $\begin{array}{c}\text { Beneficio } \\
U\end{array}$ & $\begin{array}{c}\text { Costo } \\
D=C X U\end{array}$ & $\%$ \\
\hline $\begin{array}{l}\text { Experto electromecánico en construcción } \\
\text { Estructurista } \\
\text { Obrero principal en la realización de la obra }\end{array}$ & $\begin{array}{c}1 \\
1 \\
0.1\end{array}$ & $\begin{array}{l}3.62 \\
3.62 \\
4.01\end{array}$ & $\begin{array}{c}3.62 \\
3.62 \\
0.4 \\
\end{array}$ & $\begin{array}{l}0.50 \\
0.50 \\
0.50\end{array}$ & $\begin{array}{l}1.81 \\
1.81 \\
0.20\end{array}$ & $\begin{array}{l}8.38 \% \\
8.38 \% \\
0.93 \%\end{array}$ \\
\hline
\end{tabular}

Subtotal X

\begin{tabular}{|c|c|c|c|c|c|c|}
\hline & & 3. Equipo & & & & \\
\hline Detalle & $\begin{array}{c}\text { Valor } \\
\mathrm{A}\end{array}$ & $\begin{array}{c}\text { Precio } \\
\text { B } \\
\end{array}$ & $\begin{array}{l}\text { Valor hora } \\
\mathrm{C}=\mathrm{A} \times \mathrm{B}\end{array}$ & $\begin{array}{c}\text { Beneficio } \\
U\end{array}$ & $\begin{array}{c}\text { Costo } \\
\mathrm{D}=\mathrm{C} \times \mathrm{U}\end{array}$ & $\%$ \\
\hline Instrumento de característica menor & 1 & 1.75 & 1.75 & 0.5 & 0.88 & $4 \%$ \\
\hline \multirow{2}{*}{$\begin{array}{l}\text { Equipo cortador de hierro } \\
\text { Subtotal Y }\end{array}$} & 1 & 12.5 & 12.5 & 0.5 & 6.25 & $29 \%$ \\
\hline & & & & & 7.13 & $33 \%$ \\
\hline \multicolumn{7}{|c|}{ 4. Transporte } \\
\hline & & Unidad & $\begin{array}{c}\text { Valor } \\
\mathbf{A}\end{array}$ & $\begin{array}{c}\text { Precio Unitario } \\
\text { B }\end{array}$ & $\begin{array}{c}\text { Costo } \\
\mathrm{C}=\mathrm{AX} \mathrm{B}\end{array}$ & \multirow{3}{*}{$\begin{array}{c}\% \\
100 \%\end{array}$} \\
\hline \begin{tabular}{|l} 
Subtotal Z \\
\end{tabular} & & & & & 0 & \\
\hline \multirow{3}{*}{ 2 } & & & $\begin{array}{l}\text { Total, costos directos } \\
(\mathrm{W}+\mathrm{X}+\mathrm{Y}+\mathrm{Z})\end{array}$ & & 21.6 & \\
\hline & & & $\begin{array}{l}\text { Indirectos y } \\
\text { Utilidades }\end{array}$ & $15 \%$ & 3.24 & \\
\hline & & & $\begin{array}{l}\text { Otros Indirectos: } \\
\text { Costo Total del Rubro }\end{array}$ & $0 \%$ & 24.84 & \\
\hline
\end{tabular}

En consecución con los procesos desarrollados, se desprende de manera concreta las actividades que han incidido en la producción de la obra consolidada en la tabla 3, sintetizada en el centro de costos para entender su operatividad de manera meticulosa.

\section{Tabla 3.}

Estudio de los costos por actividad.

Centro de Costos

\begin{tabular}{|c|c|c|c|c|}
\hline Detalle & Unidad & Cantidad & $\begin{array}{c}\text { V. } \\
\text { Unitario }\end{array}$ & V. Total \\
\hline \multicolumn{5}{|l|}{ Estimación presupuestaria de la obra } \\
\hline \multicolumn{5}{|l|}{ Planteamiento de terreno } \\
\hline Planeamiento y nivelación del terreno & $\mathrm{m} 2$ & 320 & 5,50 & 1760,00 \\
\hline Excavación y relleno manual & m3 & 150 & 22,00 & 3300,00 \\
\hline Excavación manual para emplazamientos sanitarias & $\mathrm{m} 3$ & 86 & 10,00 & 860,00 \\
\hline Relleno con material (mejoramiento) & m3 & 99 & 28,00 & 2772,00 \\
\hline Compactamiento de terreno & m3 & 80 & 6,50 & 520,00 \\
\hline Total, valores generales & & & & 9212,00 \\
\hline
\end{tabular}


CIENCIAMATRIA

Revista Interdisciplinaria de Humanidades, Educación, Ciencia y Tecnología

Año VI. Vol. VI. N². Edición Especial II. 2020

Hecho el depósito de ley: pp201602FA4721

ISSN-L: 2542-3029; ISSN: 2610-802X

Universidad Nacional Experimental Francisco de Miranda (UNEFM). Santa Ana de Coro. Venezuela

\section{Laura Verónica Riera-Peralta; Patricio Esteban Mendieta-Andrade; Priscila Elizabeth Muñoz-Castro}

\begin{tabular}{|c|c|c|c|c|}
\hline Armazón & & & & \\
\hline Aprovisionamiento y realización de: replantillo de piedra e $=0,18 \mathrm{~m}$ & $\mathrm{~m} 2$ & 120 & 60,00 & 7200,00 \\
\hline Producción acoplamiento de: hormigón simple sobre replantillo de piedra f' c = $192 \mathrm{~kg} / \mathrm{cm} 2$ & m3 & 10,6 & 220,00 & 2332,00 \\
\hline Producción y acoplamiento de: hormigón simple plintos f'c = 224 kg/cm2 & m3 & 6,35 & 180,00 & 1143,00 \\
\hline Aprovisionamiento y acoplamiento de: encofrados & $\mathrm{m} 2$ & 34 & 16,80 & 571,20 \\
\hline Aprovisionamiento y acoplamiento de: hierro para la estructura f'y=5.001 kg/cm2 & $\mathrm{kg}$ & 311 & 4,50 & 1399,50 \\
\hline Producción y acoplamiento de: enlucidos & $\mathrm{m} 2$ & 56 & 7,50 & 420,00 \\
\hline Aprovisionamiento y acoplamiento de: platinas base $230 \mathrm{~mm} \times 162 \mathrm{~mm} \times 3 \mathrm{~mm}$ soldadas a las zapatas & $\mathrm{u}$ & 49 & 17,00 & 833,00 \\
\hline Aprovisionamiento y acoplamiento de: bloques mk de cemento $39 \mathrm{~cm} \times 12 \mathrm{~cm}$ & $\mathrm{~m} 2$ & 79 & 50,22 & 3967,38 \\
\hline Aprovisionamiento y acoplamiento de: columna metálica $2.5 \mathrm{~g} 111 \times 52 \times 18 \times 3 \mathrm{~mm}$ & $\mathrm{~m}$ & 38 & 38,00 & 1444,00 \\
\hline Aprovisionamiento y acoplamiento de: columna metálica; 3 tubo mecánico rectangular $22 \mathrm{~mm} \times 50 \mathrm{~mm}$ & $\mathrm{~m}$ & 66 & 65,45 & 4319,70 \\
\hline Aprovisionamiento y acoplamiento de: riel de base metálica para pared rectangular $22 \mathrm{~mm} \times 50 \mathrm{~mm}$ & $\mathrm{~m}$ & 49,15 & 9,00 & 442,35 \\
\hline Aprovisionamiento y acoplamiento de: refuerzo metálico horizontal para terminación de pared $2 \mathrm{~g} 65 \times 33$ & $\mathrm{~m}$ & 49,15 & 58,00 & 2850,70 \\
\hline Total, armazón & & & & 26922,83 \\
\hline \multicolumn{5}{|l|}{ Terminados } \\
\hline Aprovisionamiento y acoplamiento de cerámica para sanitarios & $\mathrm{m} 2$ & 56 & 7,8 & 436,8 \\
\hline Aprovisionamiento y acoplamiento de puerta central metálica de $0,93 \times 2,5 \mathrm{mt}$. & $\mathrm{u}$ & 1 & 250 & 250 \\
\hline Aprovisionamiento y acoplamiento de puerta de chonta para el interior $0,85 \times 2,5 \mathrm{mt}$. & $\mathrm{u}$ & 2 & 180 & 360 \\
\hline Aprovisionamiento y acoplamiento de puerta de chonta para los baños de $0,83 \times 2,5 \mathrm{mt}$. & $\mathrm{u}$ & 1 & 185 & 185 \\
\hline Aprovisionamiento y acoplamiento de chapa & $\mathrm{u}$ & 2 & 36 & 72 \\
\hline Aprovisionamiento y acoplamiento de chapa para baños & $\mathrm{u}$ & 1 & 29 & 29 \\
\hline Aprovisionamiento e montaje de ventanas de aluminio con vidrio & $\mathrm{m} 2$ & 19 & 126,12 & 2396,28 \\
\hline $\begin{array}{l}\text { Aprovisionamiento y acoplamiento de: refuerzo metálico para pared de cemento para base de ventanas } 3 \text { tubos } \\
\text { mecánico rectangular } 20 \mathrm{~mm} \times 50 \mathrm{~mm}\end{array}$ & $\mathrm{~m}$ & 18,76 & 29,15 & 546,854 \\
\hline Total, terminados & & & & 4275,93 \\
\hline \multicolumn{5}{|l|}{ Cubierta } \\
\hline Aprovisionamiento y acoplamiento de estructura metálica 83 x 44 x 156 × 3mm. & $\mathrm{ml}$ & 78,34 & 12,14 & 951,05 \\
\hline Aprovisionamiento y acoplamiento de estructura metálica $70-34-11-3 \mathrm{~mm}$. & $\mathrm{ml}$ & 61 & 8,65 & 527,65 \\
\hline Aprovisionamiento y acoplamiento de techo $2,4 \times 1$ & $\mathrm{u}$ & 40 & 21,22 & 848,80 \\
\hline Aprovisionamiento y acoplamiento de cumbas para cubierta & $\mathrm{ml}$ & 9 & 5,2 & 46,80 \\
\hline Total, cubierta & & & & 2374,30 \\
\hline \multicolumn{5}{|l|}{ Instalaciones eléctricas } \\
\hline Aprovisionamiento e instalación de alumbrado $3 \times 33 w$ - tubos $t 6$ & $\mathrm{u}$ & 7 & 1 & 7,00 \\
\hline Aprovisionamiento e instalación de interruptor simple, 12 a, 120v & $\mathrm{u}$ & 5 & 18,12 & 90,60 \\
\hline Aprovisionamiento e instalación de interruptor doble, $12 \mathrm{a}, 120 \mathrm{v}$ & $\mathrm{u}$ & 2 & 19,2 & 38,40 \\
\hline Aprovisionamiento e instalación de tomacorriente doble, $156 \mathrm{a}, 120 \mathrm{v}$ & $\mathrm{u}$ & 11 & 39,55 & 435,05 \\
\hline Aprovisionamiento y cableado $15 \mathrm{awg} \mathrm{cu} \mathrm{-} \mathrm{por} \mathrm{ducto} \mathrm{en} \mathrm{emt} \mathrm{1/2"}$ & $\mathrm{m}$ & 99,14 & 1,76 & 174,49 \\
\hline Aprovisionamiento y cableado $17 \mathrm{awg} \mathrm{cu}$ - por ducto en emt 1/2" & $\mathrm{m}$ & 89,1 & 1,65 & 147,02 \\
\hline Aprovisionamiento y cableado $13 \mathrm{awg}$ cu - por ducto en emt 3/4" & $\mathrm{m}$ & 42 & 2,54 & 106,68 \\
\hline Interruptor monofásico hasta 40 amp & $\mathrm{u}$ & 12 & 11,3 & 135,60 \\
\hline Total, instalaciones eléctricas & & & & 1134,83 \\
\hline \multicolumn{5}{|l|}{ Instalaciones sanitarias y agua potable } \\
\hline Aprovisionamiento e instalación de duchas eléctricas, incluye: llave, ducha, rejilla. & $\mathrm{u}$ & 2 & 67,00 & 134,00 \\
\hline Aprovisionamiento e instalación de inodoro & $\mathrm{u}$ & 3 & 168,35 & 505,05 \\
\hline Aprovisionamiento e instalación de lavamanos & $\mathrm{u}$ & 3 & 140,45 & 421,35 \\
\hline Aprovisionamiento e instalación de lavaplatos & $\mathrm{u}$ & 1 & 128,45 & 128,45 \\
\hline Aprovisionamiento e instalación de tubería sanitaria y accesorios & glob & 29,5 & 68,33 & 2015,74 \\
\hline Aprovisionamiento e instalación de componentes de agua potable & glob & 2 & 88,76 & 177,52 \\
\hline Aprovisionamiento e instalación de tubería sanitaria y componentes $ø$ 3"con picada y resanada pared & glob & 2 & 126,34 & 252,68 \\
\hline Aprovisionamiento e instalación de: tubería sanitaria y componentes $\varnothing 6 "$ con ubicación y fijación pared & glob & 2 & 114,32 & 228,64 \\
\hline Aprovisionamiento e instalación de tubería pvc. $\varnothing 4$ & $\mathrm{ml}$ & 4,8 & 3,45 & 16,56 \\
\hline Aprovisionamiento e instalación de tubería pvc. Ø 5" & $\mathrm{ml}$ & 25 & 6,7 & 167,50 \\
\hline Aprovisionamiento e instalación de caja de revisión de $0,75 \times 0,75 \times 0,75 \mathrm{mt}$. & $\mathrm{u}$ & 1 & 68,5 & 68,50 \\
\hline Total, instalaciones sanitarias y agua potable & & & & 4115,99 \\
\hline Total, construcción de vivienda & & & & 48035,88 \\
\hline
\end{tabular}




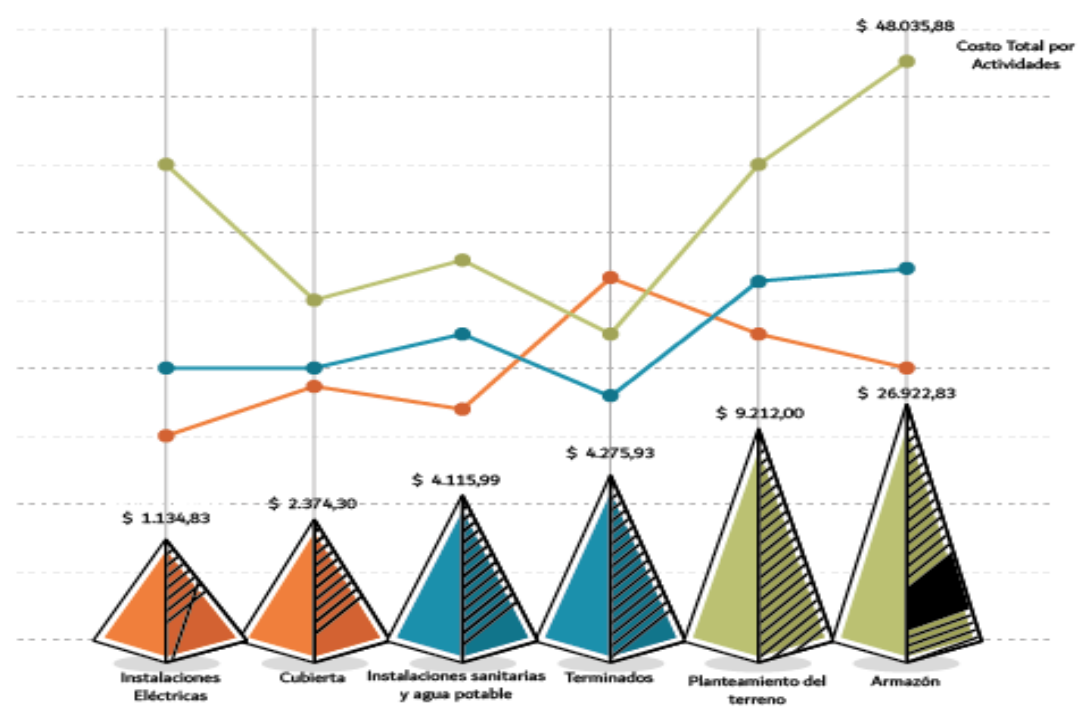

Figura 4. Gráfico de costos por actividades.

A partir del contexto de un control de costos basado en las actividades ABC cuya acción radica en el análisis de costos específicos relacionados con cada actividad, se realiza una observación técnica con el objeto de especificar el margen de rentabilidad en analogía con el mecanismo empírico, de lo cual, al obtener información precisa sobre los resultados se demuestran la existencia de utilidad reflejado en la tabla 2 que en relación al costo empírico los costos $A B C$ disminuyen el precio de venta e incrementan la rentabilidad demostrando que al utilizar un control de costos $A B C$ gana tanto el empresario como los clientes, lo que se convierte en un ganar-ganar.

\section{Tabla 3.}

Determinación de rentabilidad. 
Universidad Nacional Experimental Francisco de Miranda (UNEFM). Santa Ana de Coro. Venezuela

Laura Verónica Riera-Peralta; Patricio Esteban Mendieta-Andrade; Priscila Elizabeth Muñoz-Castro

\begin{tabular}{lrr} 
Obra (Vivienda) & & \\
\hline & Empírico & ABC \\
\hline Costo & $58.000,00$ & $48.035,88$ \\
Precio de Venta & $73.000,00$ & $66.300,00$ \\
Utilidad Bruta & $15.000,00$ & $18.264,12$ \\
CIF (cargados al gasto) & $6.316,11$ & $9.526,75$ \\
Utilidad Neta & $8.683,89$ & $8.737,37$ \\
\hline
\end{tabular}

\section{CONCLUSIÓN}

Se ha establecido en el presente trabajo de investigación que la economía de un país como el caso de la ecuatoriana siempre va a depender del sector productivo, es así que, las empresas constructoras no podían quedarse de lado, al formar parte del sector económico, dada la importancia que tienen la productividad, competitividad y el empleo en sus mecanismos con el fin de optimizar los recursos, mejorar los costos y posesionarse en el mercado que se visibiliza gracias a la aplicación de los procedimientos contables conforme la magnitud que los caracteriza.

De esta manera, en relación con el objetivo diseñado conforme al tema en estudio, en concordancia con los resultados provenientes de las encuestas realizadas a las empresas constructoras se ratifica que el régimen de los costos de producción configura una tarea diaria que deben fomentarse en todas las empresas enmarcadas en estas actividades, que para lograr sus metas y objetivos trazados en su proceso productivo, requieren de la implementación de un modelo de control de costos en el marco del control por actividades.

En consecuencia, este control deberá ser acorde a las actividades que se encuentran en constante variación dada su naturaleza, pues con esta implicación, se logra alcanzar un nivel de eficiencia en el control de sus proyectos y en la optimización de los recursos, en la medida que, el empleo de un patrón de control de costos de producción en la construcción, se convierte en un factor primordial en las empresas de la construcción, ya que dicha adecuación, no solo, permite un perfeccionamiento en el ámbito económico y 
Laura Verónica Riera-Peralta; Patricio Esteban Mendieta-Andrade; Priscila Elizabeth Muñoz-Castro competitivo, sino también, se constituye en una herramienta indispensable a la hora de tomar las decisiones de la gestión empresarial.

Es así que, en esta perspectiva y como una solución a la problemática existente, será necesario fomentar en las empresas constructoras que carecen de un contenido informativo y confiable en los procesos productivos, un control de costos relacionado con sus actividades a través de la aplicación de componentes modificadores de la estructura empresarial que permitirán mantener una información económica de primera mano y, de esta manera, la adopción de decisiones con acierto previo a los razonamientos.

Lo expuesto conlleva a que los controles contables de costos, basados en el mejoramiento de sus procesos, puedan adaptarse a las necesidades reales de cada empresa, con el fin de eliminar los errores incurridos, perfeccionar la calidad de la producción y, sobre todo reducir los costos, logrando con esto personalizar la determinación de los beneficios que se obtienen o las pérdidas que se han producido. De ahí que, en este caso en particular se deberá ejecutar las medidas correctivas, de acuerdo, a las herramientas que proporciona la contabilidad moderna, consolidada como una herramienta de análisis que permite la interpretación, el control y vigilancia de la gestión productiva de forma eficaz.

\section{REFERENCIAS CONSULTADAS}

Arcudia-Abad, C. E., Pech-Pérez, J., \& Álvarez-Romero, S. (2005). La empresa constructora y sus operaciones bajo un enfoque de sistemas. [The construction company and its operations under a systems approach]. Arcudia et al. / Ingeniería 9-1, 25-36.

Arias-Cubillo, C., \& Lopez-Lopez, A. M. (2010). Propuesta de un modelo de costos por procesos con la metodología de costos $A B C$ en una empresa del sector de la construcción. [Proposal of a process cost model with the ABC cost methodology in 
Laura Verónica Riera-Peralta; Patricio Esteban Mendieta-Andrade; Priscila Elizabeth Muñoz-Castro

a company in the construction sector]. Santiago de Cali, Colombia: Universidad de San Buenaventura Cali. Obtenido de https://url2.cl/7Pqqi

Brimson, J. (1997). Contabilidad por actividades: Un enfoque de costes basado en las actividades. [Activity-Based Accounting: An Activity-Based Cost Approach]. México: Alfa y omega grupo editor S.A. Obtenido de https://url2.cl/ha8s8

Catalá-Alís, J., \& Yepes-Piqueras, V. (1999). Aplicación del sistema de costes ABC en la gestión de proyectos $\mathrm{y}$ obras. [Application of the $\mathrm{ABC}$ cost system in the management of projects and works]. Forum Calidad, (102), 42-47.

Chang-Saltos, G. (Mayo de 2017). Tendencias del mercado de la construcción en QuitoEcuador. [Construction market trends in Quito-Ecuador]. Barcelona, España: Universitat Politécnica de Catalunya.

Cuevas, C. F., Chavez, G., Castillo, J. A., Caicedo, N. M., \& Solarte, W. F. (Julio de 2004). Costeo $A B C$ ¿Por qué y como implantarlo? [ABC Costing Why and how to implement it?]. Estudios Gerenciales(92), 47-103. Obtenido de https://url2.cl/r9ale

Galarza-Pomaquiza, M. G., Narváez-Zurita, C. I., \& Erazo-Álvarez, J. C. (2019). Costeo ABC /ABM como herramienta de control en la gestión empresarial de la Organización de Pueblos de Chibuleo (ONOPUCH). [Abc Cost /ABM as a control tool in the business management of the Organization]. Revista Arbitrada Interdisciplinaria KOINONIA, 4(2), 114-146. http://dx.doi.org/10.35381/r.k.v4i2.469

García, T., Galo, H., \& Villafuerte, O. (2015). Las restricciones al financiamiento de las PYMES del Ecuador y su incidencia en la política de inversiones. [Restrictions on financing for SMEs in Ecuador and their impact on investment policy]. Actualidad contables FACES, 49-73.

González-Pesantez, G., \& Muñoz-Delgado, C. (2010). Costos aplicados a la construcción de condominios caso práctico "LA RIOJA". [Costs applied to the construction of condominiums practical case "LA RIOJA"]. Cuenca, Azuay, Ecuador: Universidad de Cuenca. Obtenido de https://url2.cl/8ZT2S

Guerra, M., \& Yero, Y. (2009). Contabilidad por actividades. [Activity accounting]. La Habana: Ediciones Universales. Obtenido de https://url2.cl/THPV9

Hicks, D. (1998). El sistema de costos basado en las actividades (ABC): Guía para su implementación en pequeñas y medianas empresas. [The activity-based cost system (ABC): Guide for its implementation in small and medium-sized companies]. México: Alfaomega. Obtenido de https://url2.cl/fHpUq 
Laura Verónica Riera-Peralta; Patricio Esteban Mendieta-Andrade; Priscila Elizabeth Muñoz-Castro

Horngren, C., Srikant, D., \& Foster, G. (2007). Contabilidad de costos. Un enfoque gerencial [Cost accounting. A managerial approach] (Décimosegunda ed.). México: Pearson educación. Obtenido de https://url2.cl/Medew

Jiménez-Rodríguez, N. V., \& Toala-García, J. M. (Septiembre de 2014). Diseño e implementación de un sistema de costeo por órdenes de trabajo para mejorar la productividad de la constructora COPROBRA S.A. [Design and implementation of a work order costing system to improve the productivity of Cosntructora Coprobra S.A.]. Guayaquil, Guayas, Ecuador: Universidad Politécnica Salesiana. Obtenido de https://url2.cl/YygS7

Kaplan, R., \& Cooper, R. (2000). Coste y efecto. [Cost and effect]. Barcelona: Editorial Gestión. Obtenido de https://url2.cl/LSRev

Lindao-Bravo, D. S., Narváez-Zurita, C. I., \& Erazo-Álvarez, J. C. (2019). La contabilidad de gestión estratégica como herramienta multidisciplinar de planificación y control en la exportadora bananera Novamerc S.A. [Strategic management accounting as a multidisciplinary planning and control tool in the banana exporter Novamerc. Revista Arbitrada Interdisciplinaria Koinonía, 4(2), 147-182. http://dx.doi.org/10.35381/r.k.v4i2.470

Maldonado-Borja, J. D. (2017). Modelo de negocios para la creación de una empresa dedicada a la rehabilitación y optimización de espacios interiores e infraestructura de viviendas en la ciudad de Quito.[Business model for the creation of a company dedicated to the rehabilitation]. Quito, Pichincha, Ecuador: Universidad internacional del Ecuador Facultad de Ciencias Administrativas Modalidad distancia. Obtenido de https://url2.cl/vS6Rb

Mogrovejo-Juela, A. A., Narváez-Zurita, C. I., \& Erazo-Álvarez, J. C. (2019). Factores determinantes del costeo $A B C A B M$ en la industria de textiles. Caso: Fábrica Textil Multijeans. [Determining factors of $A B C A B M$ costing in the textile industry. Case: Textile Factory Multijeans]. Revista Arbitrada Interdisciplinaria KOINONIA, 4(2), 214-240. http://dx.doi.org/10.35381/r.k.v4i2.473

Morillo-Moreno, M. C. (2003). La Contabilidad de Costos y El Diseño de Mezcla de Productos. [Cost Accounting and Product Mix Design]. Actualidad contable, 1-18. Obtenido de https://url2.cl/zhsC6

Noguera-López, M. Y. (2005). Presupuestación, determinación y gestión de los costos de producción en el sector de la construcción, empresa construcciones y asfaltos 
Laura Verónica Riera-Peralta; Patricio Esteban Mendieta-Andrade; Priscila Elizabeth Muñoz-Castro

Andes C.A. [Budgeting, determination and management of production costs in the construction sector]. Bogotá, Colombia: Universidad de los Andres.

Ordoñez-Coronado, J. S. (2012). Actividad constructora en el Ecuador: Enfoque del diamanta de competitividad de M. Porte (2000-2010). [Construction activity in Ecuador: Approach to M. Porte's competitiveness diamond (2000-2010)]. Quito, Pichincha, Ecuador: Pontificia Universidad Católica del Ecuador Facultad de Economía.

Ortega-Godoy, M. A. (2006). Identificación de actividades para el costeo ABC. Seminario para optar al título de ingeniero en información y control de la gestión. [Seminar to apply for the title of engineer in information and management control]. Santiago, Chile: Universidad de Chile.

Rodríguez-Mañay, L. O., Saltos-Chacán, M. Y., \& Muñoz-Moreta, E. R. (2016). Cálculo de un cost driver para determinar el costo de una actividad empleando un modelo de Regresión Lineal o un modelo de Regresión Múltiple. [Calculation of a cost driver to determine the cost of an activity using]. Revista Publicando, 239-255.

Salinas-Bonilla, G. V. (2012). Los costos de producción y su efecto en la rentabilidad de la planta fibra de vidio en Cepolfi Industrial C.A. de la ciudad de Ambato. [Production costs and their effect on the profitability of the fiberglass plant at Cepolfi Industrial C.A. from the city]. Ambato, Tungurahua, Ecuador: Universidad Técnica de Ambato.

Sierra, R. (2002). Costos y finanzas para la toma de decisiones del mercadeo. [Costs and finances for marketing decision making]. Diario Frontera. Obtenido de https://url2.cl/twE9C

Torres- Salinas, A. (2010). Contabilidad de Costos. [Cost accounting]. México: Mc Graw Hill Editorial Interamericana S.A.

Viamontes, M., Utra, M., \& Cruz, Y. (2000). Propuesta metodológica para aplicar la contabilidad gerencial [Methodological proposal to apply managerial accounting]. Madrid: Editorial Pearson Prentice Hall. 
CIENCIAMATRIA

Revista Interdisciplinaria de Humanidades, Educación, Ciencia y Tecnología

Año VI. Vol. VI. N². Edición Especial II. 2020

Hecho el depósito de ley: pp201602FA4721

ISSN-L: 2542-3029; ISSN: 2610-802X

Universidad Nacional Experimental Francisco de Miranda (UNEFM). Santa Ana de Coro. Venezuela

Laura Verónica Riera-Peralta; Patricio Esteban Mendieta-Andrade; Priscila Elizabeth Muñoz-Castro 